\title{
Dephasing in strongly anisotropic black phosphorus
}

\author{
N. Hemsworth, ${ }^{1}$ V. Tayari, ${ }^{1}$ F. Telesio, ${ }^{2}$ S. Xiang, ${ }^{2}$ S. Roddaro, ${ }^{2}$ M. Caporali, ${ }^{3}$ A. Ienco, ${ }^{3}$ M. Serrano-Ruiz, ${ }^{3}$ M. Peruzzini, ${ }^{3}$ \\ G. Gervais,,$^{4, *}$ T. Szkopek, ${ }^{1}$ and S. Heun ${ }^{2}$ \\ ${ }^{1}$ Department of Electrical and Computer Engineering, McGill University, Montréal, Québec, H3A 2A7, Canada \\ ${ }^{2}$ NEST, Istituto Nanoscienze-CNR and Scuola Normale Superiore, I-56127 Pisa, Italy \\ ${ }^{3}$ Istituto Chimica dei Composti OrganoMetallici-CNR, I-50019 Sesto Fiorentino, Italy \\ ${ }^{4}$ Physics Department, McGill University, Montréal, Québec, H3A 2T8, Canada
}

(Received 24 August 2016; revised manuscript received 5 November 2016; published 2 December 2016)

\begin{abstract}
Weak localization was observed in a black phosphorus field-effect transistor $65 \mathrm{~nm}$ thick. The weak localization behavior was found to be in excellent agreement with the Hikami-Larkin-Nagaoka model for fields up to $1 \mathrm{~T}$, from which characteristic scattering lengths could be inferred. The temperature dependence of the phase coherence length $L_{\varphi}$ was investigated, and above $1 \mathrm{~K}$, it was found to decrease weaker than the $L_{\varphi} \propto T^{-1 / 2}$ dependence characteristic of electron-electron scattering in the presence of elastic scattering in two dimensions. Rather, the observed power law was found to be close to that observed previously in quasi-one-dimensional systems such as metallic nanowires and carbon nanotubes.
\end{abstract}

DOI: 10.1103/PhysRevB.94.245404

\section{INTRODUCTION}

The recent surge of interest in the field of two-dimensional (2D) atomic crystals has led to a number of important advances in our understanding of solid-state physics in two dimensions. These include transition metal dichalcogenides, topological insulators such as bismuth selenide, and phosphorene. For the latter, its underlying crystal structure inherited from black phosphorus (bP) is a "puckered" honeycomb lattice hosting one-dimensional chains of atoms displaced out of the atomic plane of the honeycomb lattice, and running along the zigzag axis [see Fig. 1(a)]. Few-layer bP has been the subject of recent investigations wherein strong anisotropy was observed in electronic transport, optical absorption, thermal conductivity, and angle-resolved photoemission measurements [1-4]. Here, we have studied the weak localization in a bP field-effect transistor (FET) and found that the phase coherence length decays with temperature at a slower rate than that expected for a $2 \mathrm{D}$ material. This more robust coherence is reminiscent to that previously reported in quasi-one-dimensional systems such as nanotubes [5] and metallic nanowires [6]. Our experimental study of weak localization in bP is amongst very few investigations of weak localization in strongly anisotropic media.

Unlike semimetallic graphene, bP is a direct gap semiconductor [7,8]. The bandgap is $0.3 \mathrm{eV}$ in bulk and increases by quantum confinement to $1-2 \mathrm{eV}$ in the monolayer limit [9-11], ideal for applications in electronics and optoelectronics [12]. Black phosphorus FETs have been demonstrated by various groups [1,13-17], with hole field-effect mobilities reaching up to $1350 \mathrm{~cm}^{2} / \mathrm{Vs}$ and $10^{5}$ current modulation at room temperature [18].

In a disordered 2D system, coherent backscattering of charge carriers gives rise to a peak in the magnetoresistance known as weak localization (WL); see [19] for a review. Previous work on WL in 2D crystals has focused primarily on graphene [20-22]. Weak (anti)localization was also observed

*gervais@physics.mcgill.ca in novel topological phases of matter such as topological insulators [23-25], Dirac semimetals [26], and HgTe quantum wells [27]. Recently WL has been observed in molybdenum disulfide, revealing a phase coherence length $L_{\varphi}$ of $\sim 50 \mathrm{~nm}$ at $400 \mathrm{mK}$ that decays with increasing temperature as $T^{-\alpha} \simeq$ $T^{-1 / 2}$ [28], characteristic of electron-electron interactions in two dimensions. Here, we have observed WL in a 65-nm-thick bP FET (which forms a $\sim 3$-nm-thick 2D hole gas in an accumulation layer at the $\mathrm{bP} / \mathrm{SiO}_{2}$ interface [29]) whose dephasing length exponent is found to be suppressed, and closer to a value of $1 / 3$. We attribute this WL behavior to the anisotropic nature of the puckered bP atomic crystal structure whose hole dispersion is distinct along the hard (along) and easy (against) axis of the puckers; see Fig. 1.

\section{RESULTS AND DISCUSSION}

\section{A. Carrier density and mobility}

A photograph and an atomic force microscopy image of the bP FET are shown in Figs. 1(b) and 1(c), respectively. The longitudinal resistance $R_{x x}$ versus back gate voltage $V_{g}$ is plotted in Fig. 2(a), with sample temperature $T$ as a parameter. Hole carrier density $p$ inferred from the Hall resistance, shown in Fig. 2(b) with the component symmetric in $B$ removed, is found to depend linearly on gate voltage $V_{g}$ over the range of p-type conduction $\left(V_{g}<-30 \mathrm{~V}\right)$, with a density of approximately $10^{13} / \mathrm{cm}^{2}$ at $V_{g}=-30 \mathrm{~V}$. The field-effect hole mobility, $\mu_{\mathrm{FE}}=(L / W) \partial G_{x x} / \partial\left(C_{g} V_{g}\right)$ was found to reach a peak value of $300 \mathrm{~cm}^{2} / \mathrm{Vs}$ at a gate voltage $V_{g}=-70 \mathrm{~V}$, with a negligible dependence upon temperature.

\section{B. Weak localization and fitting}

The measured WL peak in longitudinal resistance is shown in the plot of $\Delta R_{x x} / R_{x x}(0)=\left(R_{x x}(B)-R_{x x}(0)\right) / R_{x x}(0)$ versus magnetic field $B$ and gate voltage $V_{g}$ in Fig. 3(a). The amplitude of the WL peak increases with increasing hole density attaining a maximum value at the highest negative gate voltage used in this experiment, $-80 \mathrm{~V}$. The temperature dependence of the WL peak at $V_{g}=-80 \mathrm{~V}$ and $V_{g}=-40 \mathrm{~V}$ is plotted 

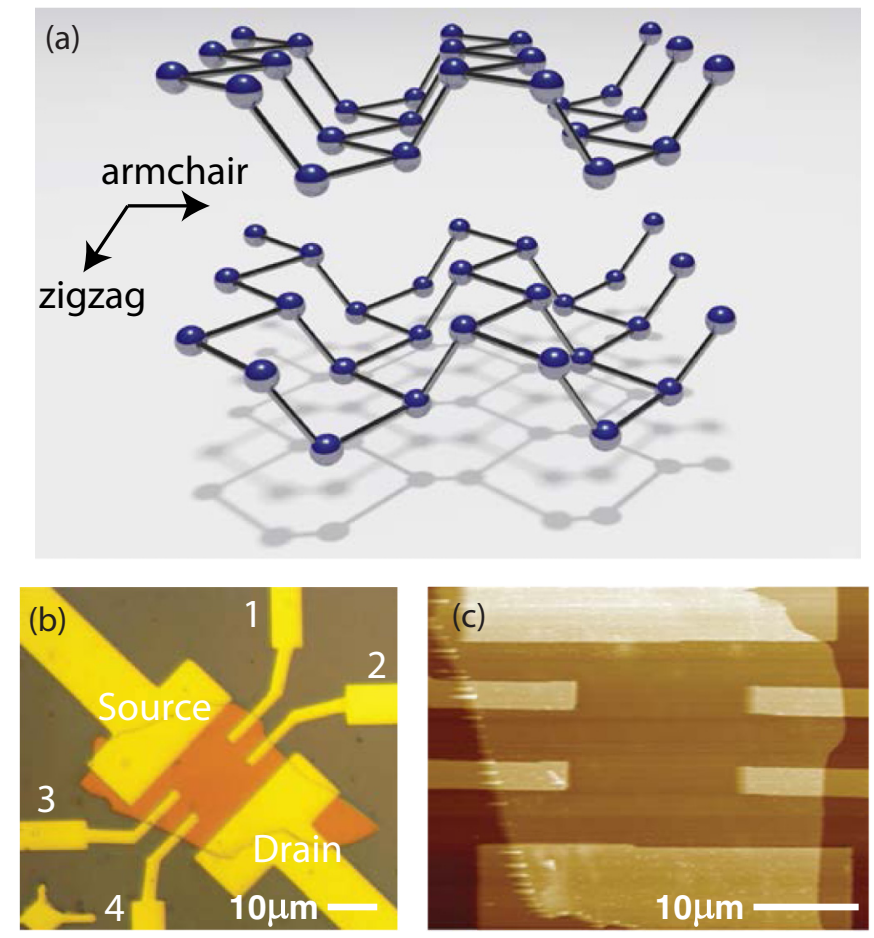

FIG. 1. Black phosphorus field-effect transistor. (a) Schematic of the puckered honeycomb crystal structure of bP. (b) Optical reflection microscopy image of the bP FET. The longitudinal resistance $R_{x x}$ was measured with voltage probes 1 and 2, and the Hall resistance $R_{x y}$ with voltage probes 1 and 3. (c) AFM image of the bP FET. The bP thickness was measured to be $65 \pm 2 \mathrm{~nm}$.
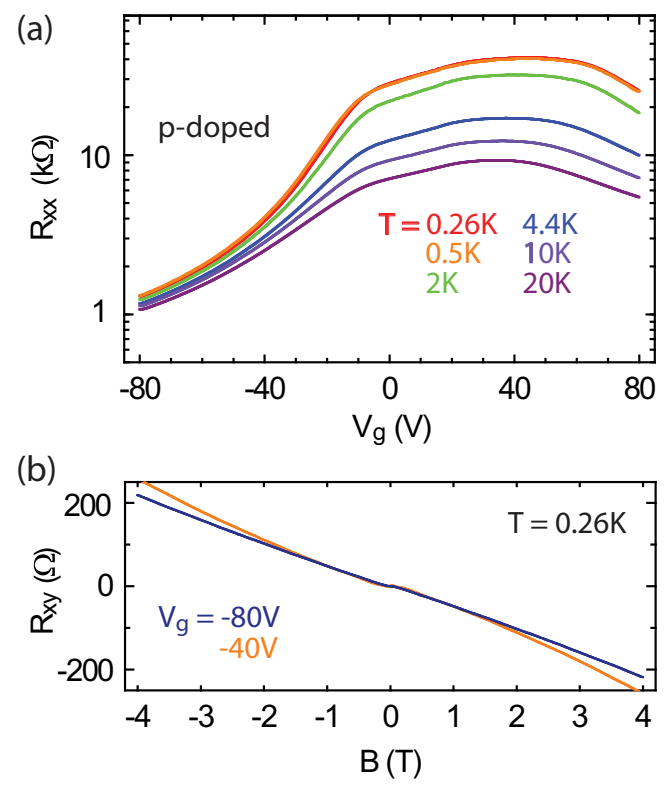

FIG. 2. Transport measurements versus backgate voltage and temperature. (a) The measured longitudinal resistance $R_{x x}$ versus gate voltage $V_{g}$, at temperatures $T=0.26 \mathrm{~K}$ to $20 \mathrm{~K}$. The region of strong p-doping is identified. (b) The Hall resistance $R_{x y}$ versus magnetic field $B$ at $T=0.26 \mathrm{~K}$, with the symmetric in $B$ component removed, as measured at $V_{g}=-80 \mathrm{~V}$ and $-40 \mathrm{~V}$.

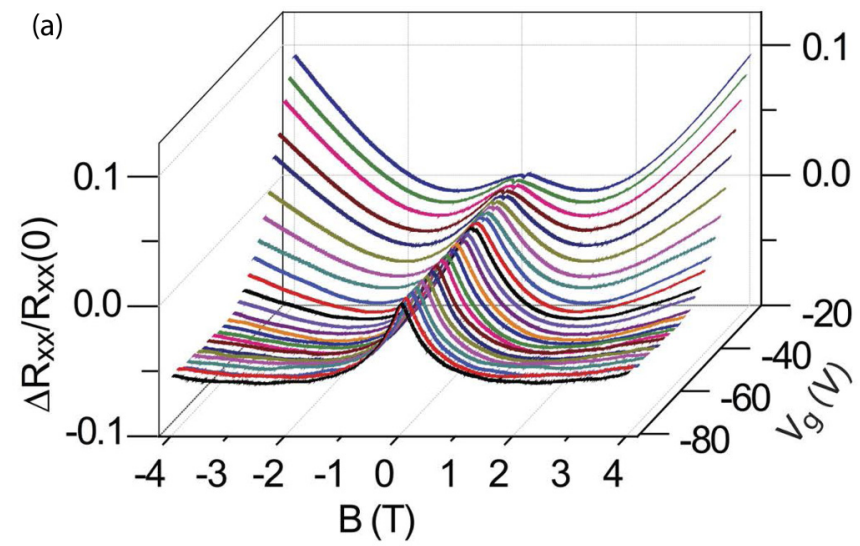

(b)

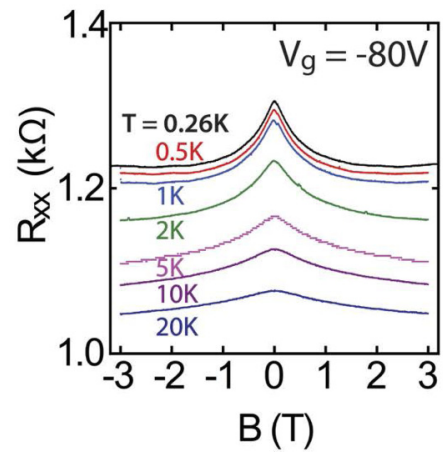

(c)

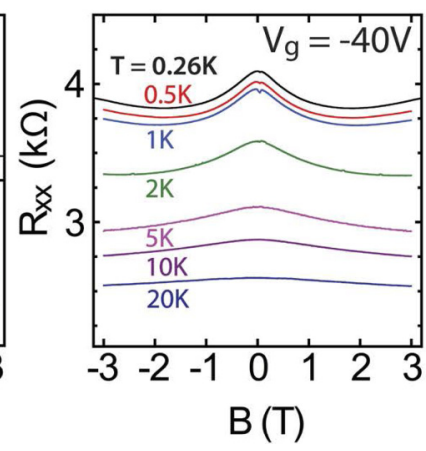

FIG. 3. Weak localization measurements. (a) A weak localization peak is observed in a plot of the normalized longitudinal resistance $\left(R_{x x}(B)-R_{x x}(0)\right) / R_{x x}(0)$ versus magnetic field $B$ and gate voltage $V_{g}$ at $T=0.26 \mathrm{~K}$. The temperature dependence of the longitudinal resistance $R_{x x}$ versus magnetic field $B$ at (b) $V_{g}=-80 \mathrm{~V}$ and (c) at $V_{g}=-40 \mathrm{~V}$.

in Figs. 3(b) and 3(c), respectively. The WL correction to the resistance decreases with increasing temperature eventually disappearing at temperatures above $20 \mathrm{~K}$, as expected.

The Hikami-Larkin-Nagaoka (HLN) theory [19,30,31] gives a quantitative prediction for the WL correction to the sheet conductance,

$$
\begin{aligned}
\Delta \sigma= & -\frac{e^{2}}{2 \pi^{2} \hbar}\left(\Psi\left(\frac{1}{2}+\frac{B_{1}}{B}\right)-\Psi\left(\frac{1}{2}+\frac{B_{2}}{B}\right)\right. \\
& \left.+\frac{1}{2} \Psi\left(\frac{1}{2}+\frac{B_{3}}{B}\right)-\frac{1}{2} \Psi\left(\frac{1}{2}+\frac{B_{2}}{B}\right)\right),
\end{aligned}
$$

where $\Psi$ is the digamma function. The field parameters in the above expression are given by $B_{1}=B_{0}+B_{s o}+B_{s}$, $B_{2}=\frac{4}{3} B_{s o}+\frac{2}{3} B_{s}+B_{\varphi}$ and $B_{3}=2 B_{s}+B_{\varphi}$ where $B_{0}, B_{s o}$, $B_{s}$, and $B_{\varphi}$ are the characteristic fields associated with elastic scattering, spin-orbit scattering, magnetic scattering, and inelastic scattering (dephasing), respectively. Note that this theory is universally valid for systems with an arbitrarily complex Fermi surface, including a strong anisotropy [32].

The measured WL peak (normalized) resistances were inverted into conductivity by the usual tensor relation $\sigma_{x x}=$ $\rho_{x x} /\left(\rho_{x x}^{2}+\rho_{x y}^{2}\right)$, with negligible contributions from the (transverse) Hall resistivity [see Fig. 2(b)]. To account for the background resistivity, the relation $\Delta \sigma=-(L / W)\left(R_{x x}(B)-\right.$ 


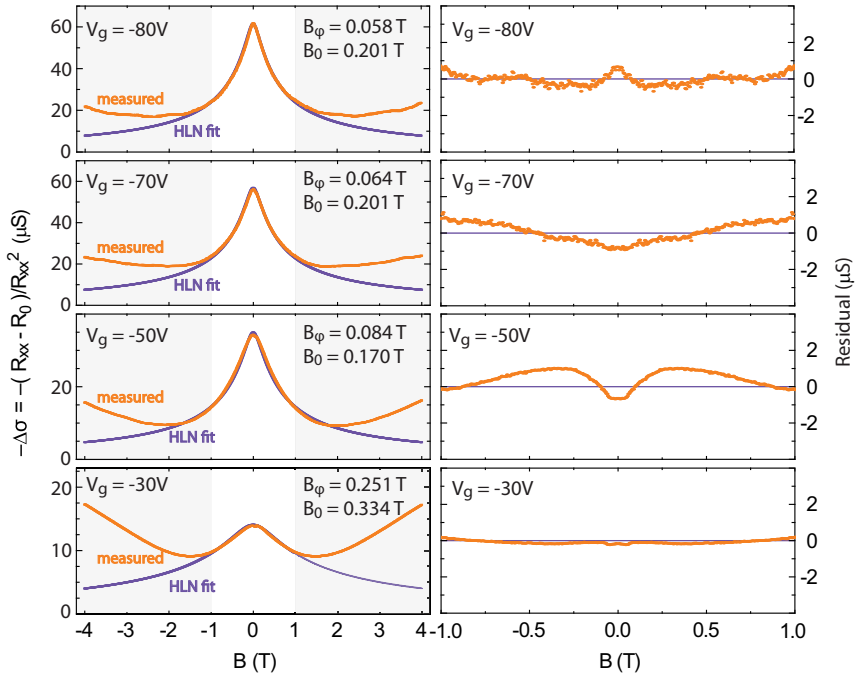

FIG. 4. HLN weak localization fits. The measured longitudinal conductance correction $-\Delta \sigma$ versus magnetic field $B$ and a best fit to a Hikami-Larkin-Nagaoka theory over the field range $-1 \mathrm{~T}<$ $B<1 \mathrm{~T}$, where weak localization dominates, is shown for various gate voltages $V_{g}$ at $T=0.26 \mathrm{~K}$. The extracted characteristic fields $B_{0}$ for elastic and $B_{\varphi}$ for inelastic (dephasing) scattering are indicated.

$\left.R_{0}\right) / R_{x x}^{2}(B)$ was used, where $R_{0}$ is the classical Drude resistance in the absence of a WL correction. The determination of the $R_{0}$ is not trivial, and was thus left as a fit parameter, however, it was verified that its trend in temperature followed that of the measured transport mobility of the bP. This WL correction to conductivity was numerically fitted to the HLN model under the approximation of negligible spin-orbit coupling, $B_{s o}=0$ (consistent with the sign of the magnetoresistance), and negligible magnetic impurity scattering, $B_{s}=0$. Both approximations are appropriate for our bP crystals [33]. Measurements and numerical fits of the WL contribution to conductivity $\Delta \sigma$ are plotted in Fig. 4 versus backgate voltage at temperature $T=0.26 \mathrm{~K}$. The fit was performed over the magnetic field range $-1 \mathrm{~T}<B<+1 \mathrm{~T}$, where the WL feature dominates the magnetoresistance. The fit quality was evaluated over the same $B$ range, with an $R^{2}$ coefficient of determination of at minimum 0.99. The residuals are also shown in the right panels of Fig. 4.

The extracted characteristic fields $B_{0, \varphi}$ are related to the scattering lengths $L_{0, \varphi}$ by considering the phase shift of diffusing charge carriers under the influence of a magnetic field, $B L^{2}=\hbar / 4 e$. From these fields, we can deduce elastic as well as inelastic (dephasing) characteristic lengths, $L_{0}$ and $L_{\varphi}$, respectively. These lengths are plotted versus gate voltage $V_{g}$ in Fig. 5(a) at $T=0.26 \mathrm{~K}$. In particular, the dephasing length $L_{\varphi}$ clearly increases with increasing hole density, reaching a maximum of $55 \mathrm{~nm}$, whereas $L_{0}$ remains nearly independent over a broad range of carrier density. We have verified that $L_{0}$ scales linearly with the transport mobility, as expected for an elastic scattering process occurring in the presence of both a phonon bath and disorder. We have also verified that the values of $L_{\varphi}$ change only marginally (less than $2 \%$ ) if we include weak magnetic impurity scattering in the HLN fit.
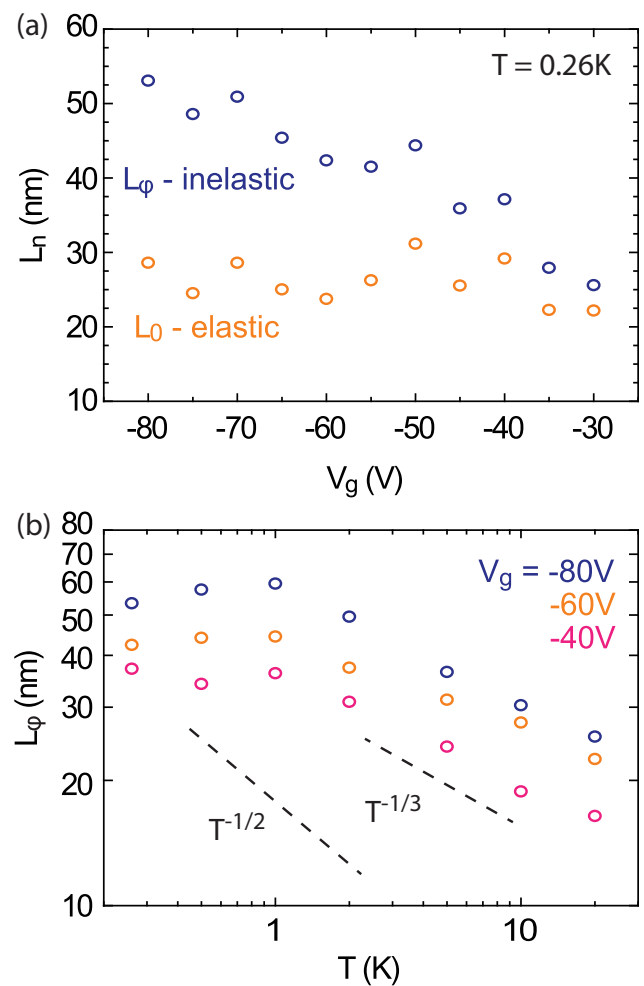

FIG. 5. Scattering lengths and power laws. (a) The inelastic scattering length $L_{\varphi}$ and elastic scattering length $L_{0}$ versus gate voltage $V_{g}$, at temperature $T=0.26 \mathrm{~K}$. (b) The inelastic scattering length $L_{\varphi}$ versus temperature $T$ at various gate voltages $V_{g}$. The $T^{-1 / 2}$ temperature dependence associated with electron-electron scattering in the 2D diffusive limit is shown for comparison, as well as a $T^{-1 / 3}$ power law.

For WL occurring in typical disordered 2D systems, the dephasing length is related to the inelastic scattering time via $L_{\varphi}^{2}=D \tau_{\varphi}$, where $D$ is the elastic diffusion coefficient. Electron-electron scattering in the absence of elastic scattering is expected to give a scattering rate $1 / \tau_{\varphi} \propto T^{2}$, and hence $L_{\varphi} \propto T^{-1}$ [34]. In the diffusive transport regime, appropriate here since $L_{0}<L_{\varphi}$, the electron-electron scattering rate is expected to follow $1 / \tau_{\varphi} \propto T$ [35-37], and hence $L_{\varphi} \propto T^{-1 / 2}$. This characteristic inelastic scattering rate has been observed in graphene [20-22,34] and $\mathrm{MoS}_{2}$ [28], however, our WL measurements in a bP thin film clearly do not follow this trend. The temperature dependence of $L_{\varphi}$ is shown in Fig. 5(b) in a $\log -\log$ plot at fixed gate voltages $V_{g}=-40,-60,-80 \mathrm{~V}$. The experimental error in $L_{\varphi}$ was determined by way of visual inspection of the quality of the fit when varying one parameter and keeping all others constant, and are contained within the size of the symbols. The saturation of $L_{\varphi}$ at temperatures below $1 \mathrm{~K}$ is most likely due to dynamical impurities such as nonmagnetic defects, as previously observed in a variety of metallic and semiconducting 2D systems [38]. However, above $1 \mathrm{~K}$ the temperature dependence of $L_{\varphi}$ does not follow the $T^{-1 / 2}$ (dashed line) behavior expected a priori from electron-electron scattering in the presence of elastic scattering in two dimensions. A weighted fit of the inelastic scattering length versus temperature rather show a suppressed dephasing length exponent (and corresponding 95\% confidence 
interval within bracket) $\alpha$ of $0.29[0.26-0.32], 0.22[0.19-$ $0.25], 0.27[0.23-0.31]$, with $R^{2}$ coefficients all greater than 0.99 at gate voltages $V_{g}=-80,-60,-40 \mathrm{~V}$, respectively.

\section{Discussion}

Our WL measurements reveal the presence of an inelastic scattering mechanism that is characterized by a decay in dephasing length weaker than $T^{-1 / 2}$. This is in contrast with previous measurements in few-layer bP films [39] where a power law was also deduced, albeit with saturation of $L_{\varphi}$ at higher temperatures than observed here $(5 \mathrm{~K}$ as opposed to $1 \mathrm{~K}$ in our case). The analysis of [39] included a fitting parameter for valley degeneracy in bP. This parameter was found to differ from unity (no valley degeneracy) and to vary from $\sim 1.2$ to 0.3 over the range of temperatures where the power law exponent for $L_{\varphi}$ was extracted. The band structure of bP has been shown by photoemission studies [13], magnetotransport [29], and density functional theory [40] to be absent of any valley degeneracies, calling into question the robustness of the $T^{-1 / 2}$ scaling reported in Ref. [39]. Consistent with this conclusion, a weaker power-law dependence $L_{\varphi} \propto T^{-0.4}$ was indeed very recently observed in another paper on few-layer bP, but nonetheless interpreted as evidence for electron-electron interactions in two dimensions as the dominant scattering mechanism [41].

A weaker loss of the dephasing length with temperature, as compared to the $2 \mathrm{D}$ case, has previously been observed in the presence of a strong one-dimensional (1D) confinement potential, e.g., in carbon nanotubes [5], and $L_{\varphi}$ was shown to be well described by the theoretical prediction of a $T^{-1 / 3}$ power law. Remarkably, the transition from the 2D limit to the quasi-1D limit was observed in the systematic study undertaken by Natelson et al. [6] whereby the dephasing time in metallic nanowires was measured as a function of the wire width down to $5 \mathrm{~nm}$. In this regime of width and low temperatures, several heuristic length scales can be estimated placing the wires well into a quasi-1D regime. In this study a lower saturation temperature was observed, however, the dephasing time $\tau_{\varphi}$ was determined to follow closely a $T^{-2 / 3}$ power law, corresponding to a dephasing length exponent $\alpha=1 / 3$. Figure 6 shows a comparison between Natelson's dephasing time and the dephasing time of our bP FET at $V_{g}=-80 \mathrm{~V}$. The expected $T^{-2 / 3}$ power law for $\tau_{\varphi}$ in the quasi-1D limit is also shown as a dotted line.

To our knowledge, there are two known scenarios for which a power-law exponent weaker than $\alpha=1 / 2$ can occur. These are (i) strong confinement due to a 1D potential, as mentioned above and (ii) dephasing by edge domain granularity [42]. The crystalline order in our bP FET was carefully investigated by way of both unpolarized and polarized micro-Raman measurements, and these data strongly suggest the bP flake to be an homogeneous single crystal (see Supplemental Material [43]) rendering the granularity scenario unlikely. It is therefore more likely that the anisotropic nature of bP arising from puckers in the atomic plane which give rise to an ellipsoidal Fermi surface with heavy hole mass in the zigzag direction parallel with the puckers, and light hole mass in the armchair direction perpendicular to the puckers. Making use of calculated values for the effective masses as reported in Ref. [40], we estimate (a)
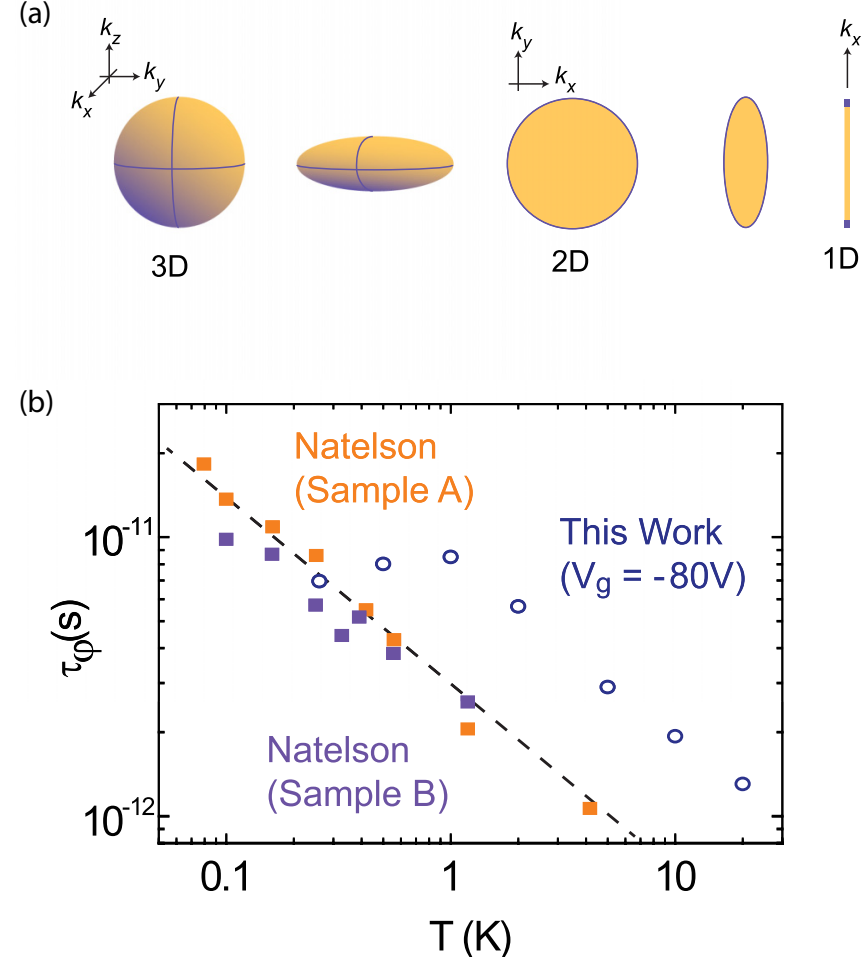

FIG. 6. Comparison with quasi-one-dimensional metallic wire. (a) Schematic depicting the Fermi surface evolution from three dimensions to one dimension. (b) The data in bP at gate voltage $V_{g}=-80 \mathrm{~V}$ converted into dephasing time $\tau_{\varphi}$ are compared with those of Natelson et al. [6] measured in metallic nanowires in the quasi-1D regime. The dotted line shows a dephasing time decaying as $T^{-2 / 3}$, i.e., corresponding to a power law exponent $\alpha=1 / 3$ for $L_{\varphi}$.

the corresponding Fermi wavelengths along and against the puckers to be $\lambda_{F, z z}=5$ and $\lambda_{F, a c}=12 \mathrm{~nm}$, respectively, at least one order of magnitude larger than any atomic length scales. In the basal direction, the presence of an electric field from the backgate has been shown to create a $2 \mathrm{D}$ hole gas with a thickness $\delta_{2 \text { deg }}$ of $\approx 3 \mathrm{~nm}[29,44]$, a scale that is much less than the thermal length $L_{T}=\sqrt{\hbar D / k_{B} T} \sim 10-60 \mathrm{~nm}$ over all temperatures investigated in our experiment. These length scales place the bP FET into a strongly anisotropic regime for which weak, and even strong localization, has not yet been well studied.

\section{CONCLUSION}

In summary, WL was observed in a 65-nm-thick black phosphorus field-effect transistor and studied over a broad range of gate voltages (hole density) and temperatures. The measured WL demonstrates a more robust coherence observed as a function of temperature similar to what was previously observed in quasi-one-dimensional systems such as nanotubes and metallic nanowires. Since it is highly sensitive to dimensionality, we attributed the more robust character of $L_{\varphi}$ in bP to the highly anisotropic nature of the puckered honeycomb crystal structure. In the future, it will be of great interest to understand exactly how dephasing is affected by strong 
anisotropy in post-graphene $2 \mathrm{D}$ atomic crystals, a subject that is currently nearly free of any theoretical knowledge.

\section{ACKNOWLEDGMENTS}

We thank Vincenzo Piazza for his help with the polarized Raman measurements. The authors thank NSERC (Canada), Cifar (Canada), the Canada CRC Program, Hydro-Québec, Institut de l'énergie Trottier, and FRQNT (Québec). We also thank the European Research Council (ERC) under the European Union's Horizon 2020 research and innovation program (Grant Agreement No. 670173). We acknowledge funding from the Italian Ministry of Foreign Affairs, Direzione Generale per la Promozione del Sistema Paese. Financial support from the CNR in the framework of the agreements on scientific collaboration between CNR and NRF (Korea), CNRS (France), and RFBR (Russia) is acknowledged. Furthermore, funding from the European Union Seventh Framework Programme under Grant Agreement No. 604391 Graphene Flagship is acknowledged. G.G. acknowledges two short-term mobility grants from CNR. S.H. acknowledges SNS Grant No. SNS16_B_HEUN.
[1] F. Xia, H. Wang, and Y. Jia, Nat. Comm. 5, 4458 (2014).

[2] X. Wang, A. M. Jones, K. L. Seyler, V. Tran, Y. Jia, H. Zhao, H. Wang, L. Yang, X. Xu, and F. Xia, Nat. Nanotech. 10, 517 (2015).

[3] Z. Luo, J. Maessen, Y. Deng, Y. Du, R. P. Garrelts, M. S. Lundstrom, P. D. Ye, and X. Xu, Nat. Comm. 6, 8572 (2015).

[4] J. Kim, S. S. Baik, S. H. Ryu, Y. Sohn, S. Park, B.-G. Park, J. Dendinger, Y. Yi, H. J. Choi, and K. S. Kim, Science 349, 723 (2015).

[5] J. Appenzeller, R. Martel, P. Avouris, H. Stahl, U. T. Hunger, and B. Lengeler, Phys. Rev. B 64, 121404(R) (2001).

[6] D. Natelson, R. L. Willett, K. W. West, and L. N. Pfeiffer, Phys. Rev. Lett. 86, 1821 (2001).

[7] R. W. Keyes, Phys. Rev. 92, 580 (1953).

[8] A. Morita, Appl. Phys. A 39, 227 (1986).

[9] Y. Du, C. Ouyang, S. Shi, and M. Lei, J. Appl. Phys. 107, 093718 (2010).

[10] S. Das, W. Zhang, M. Demarteau, A. Hoffmann, M. Dubey, and A. Roelofs, Nano Lett. 14, 5733 (2014).

[11] L. Liang, J. Wang, W. Lin, B. G. Sumpter, V. Meunier, and M. Pan, Nano Lett. 14, 6400 (2014).

[12] A. Castellanos-Gomez, J. Phys. Chem. Lett. 6, 4280 (2015).

[13] L. Li, Y. Yu, G. J. Ye, Q. Ge, X. Ou, H. Wu, D. Feng, X. H. Chen, and Y. Zhang, Nat. Nanotechnol. 9, 372 (2014).

[14] H. Liu, A. T. Neal, Z. Zhu, Z. Luo, X. Xu, D. Tomanek, and P. D. Ye, ACS Nano 8, 4033 (2014).

[15] S. P. Koenig, R. A. Doganov, H. Schmidt, A. H. Castro Neto, and B. Ozyilmaz, Appl. Phys. Lett. 104, 103106 (2014).

[16] A. Castellanos-Gomez, L. Vicarelli, E. Prada, J. O. Island, K. L. Narasimha-Acharya, S. I. Blanter, D. J. Groenendijk, M. Buscema, G. A. Steele, J. V. Alvarez, H. W. Zandbergen, J. J. Palacios, and H. S. J. van der Zant, 2D Mater. 1, 025001 (2014).

[17] J. D. Wood, S. A. Wells, D. Jariwala, K.-S. Chen, E. Cho, V. K. Sangwan, X. Liu, L. J. Lauhon, T. J. Marks, and M. C. Hersam, Nano Lett. 14, 6964 (2014).

[18] X. Chen, Y. Wu, Z. Wu, Y. Han, S. Xu, L. Wang, W. Ye, T. Han, Y. He, Y. Cai, and N. Wang, Nat. Comm. 6, 7315 (2015).

[19] G. Bergmann, Phys. Rep. 107, 1 (1984).

[20] S. V. Morozov, K. S. Novoselov, M. I. Katsnelson, F. Schedin, L. A. Ponomarenko, D. Jiang, and A. K. Geim, Phys. Rev. Lett. 97, 016801 (2006).
[21] X. Wu, X. Li, Z. Song, C. Berger, and W. A. de Heer, Phys. Rev. Lett. 98, 136801 (2007).

[22] F. V. Tikhonenko, D. W. Horsell, R. V. Gorbachev, and A. K. Savchenko, Phys. Rev. Lett. 100, 056802 (2008).

[23] M. J. Brahlek, N. Koirala, J. Liu, T. I. Yusufaly, M. Salehi, M.-G. Han, Y. Zhu, D. Vanderbilt, and S. Oh, Phys. Rev. B 93, 125416 (2016).

[24] Y. Jing, S. Huang, K. Zhang, J. Wu, Y. Guo, H. Peng, Z. Liu, and H. Q. Xu, Nanoscale 8, 1879 (2016).

[25] D. L. Mo, W. B. Wang, and Q. Cai, Nanoscale Research Letters 11, 1 (2016).

[26] B. Zhao, P. Cheng, H. Pan, S. Zhang, B. Wang, G. Wang, F. Xiu, and F. Song, Sci. Rep. 6, 22377 (2016).

[27] G. M. Minkov, A. V. Germanenko, O. E. Rut, A. A. Sherstobitov, S. A. Dvoretski, and N. N. Mikhailov, Phys. Rev. B 91, 205302 (2015).

[28] A. T. Neal, H. Liu, J. Gu, and P. D. Ye, ACS Nano 7, 7077 (2013).

[29] V. Tayari, N. Hemsworth, I. Fakih, A. Favron, E. Gaufres, G. Gervais, R. Martel, and T. Szkopek, Nat. Comm. 6, 7702 (2015).

[30] S. Hikami, A. I. Larkin, and Y. Nagaoka, Prog. Theor. Phys. 63, 707 (1980).

[31] S. Maekawa and H. Fukuyama, J. Phys. Soc. Jpn. 50, 2516 (1981).

[32] D. Rainer and G. Bergmann, Phys. Rev. B 32, 3522 (1985).

[33] We have verified that $B_{s}$ self-consistently converges to zero if used as a free fit parameter. Furthermore, we have performed an analysis of the bP crystal used in these experiments by inductively coupled plasma-mass spectrometry (ICP-MS) which revealed a negligible level of magnetic and heavy elements, below $1 \%$. In addition, phosphorus being a light element, its spin orbit coupling is negligibly small.

[34] F. V. Tikhonenko, A. A. Kozikov, A. K. Savchenko, and R. V. Gorbachev, Phys. Rev. Lett. 103, 226801 (2009).

[35] B. L. Altshuler, D. Khmelnitzkii, A. I. Larkin, and P. A. Lee, Phys. Rev. B 22, 5142 (1980).

[36] E. Abrahams, P. W. Anderson, P. A. Lee, and T. V. Ramakrishnan, Phys. Rev. B 24, 6783 (1981).

[37] B. L. Altshuler, A. G. Aronov, and D. E. Khmelnitsky, J. Phys. C 15, 7367 (1982).

[38] J. J. Lin and J. P. Bird, J. Phys.: Condens. Matter 14, R501 (2002).

[39] Y. Du, A. T. Neal, H. Zhou, and P. D. Ye, 2D Mater. 3, 024003 (2016). 
[40] J. Qiao, X. Kong, Z.-X. Hu, F. Yang, and W. Ji, Nat. Comm. 5, 4475 (2014).

[41] Y. Shi, N. Gillgren, T. Espiritu, S. Tran, J. Yang, K. Watanabe, T. Taniguchi, and C. N. Lau, 2D Mater. 3, 034003 (2016).

[42] E. Beliayev, B. Beletsev, and Y. Kolesnichenko, Low Temp. Phys. (Kharkov) 37, 318 (2011).
[43] See Supplemental Material at http://link.aps.org/supplemental/ 10.1103/PhysRevB.94.245404 for details on device fabrication and Raman characterization.

[44] L. Li, G. J. Ye, V. Tran, R. Fei, G. Chen, H. Wang, J. Wang, K. Watanabe, T. Taniguchi, L. Yang, X. H. Chen, and Y. Zhang, Nat. Nanotech. 10, 608 (2015). 